\title{
Time-Efficient Perfusion Imaging Using DCE- and DSC-MRI
}

\author{
Ondřej Macíček ${ }^{1,2}$, Radovan Jiř́k ${ }^{1}$, Jan Mikulka ${ }^{3}$, Michal Bartoš ${ }^{4}$, Andrea Šprláková-Puková ${ }^{5}$, \\ Miloš Keřkovský ${ }^{5}$, Zenon Starčuk jr. ${ }^{1}$, Karel Bartušek ${ }^{1}$ and Torfinn Taxt $^{6}$
}

${ }^{1}$ Institute of Scientific Instruments of the Czech Academy of Sciences, Královopolská, 62/147, 612 00, Brno, Czech Republic, macicek@isibrno.cz

${ }^{2}$ Department of Biomedical Engineering, The Faculty of Electrical Engineering and Communication, Brno University of Technology, Technická, 3082/12, 616 00, Brno, Czech Republic

${ }^{3}$ Department of Theoretical and Experimental Electrical Engineering, The Faculty of Electrical Engineering and Communication, Brno University of Technology, Technická, 3082/12, 616 00, Brno, Czech Republic

${ }^{4}$ Institute of Information Theory and Automation of the Czech Academy of Sciences, Pod Vodárenskou věží, 4, 182 08, Prague

8, Czech Republic

${ }^{5}$ Department of Radiology and Nuclear Medicine, The University Hospital Brno and Masaryk University, Jihlavská, 340/20, 625 00, Brno, Czech Republic

${ }^{6}$ The Department of Biomedicine, Faculty of Medicine, University of Bergen, Jonas Lies vei, 91, 5009, Bergen, Norway

\begin{abstract}
Dynamic contrast enhanced MRI (DCE-MRI) and dynamic susceptibility contrast MRI (DSC-MRI) are perfusion imaging techniques used mainly for clinical and preclinical measurement of vessel permeability and capillary blood flow, respectively. It is advantageous to apply both methods to exploit their complementary information about the perfusion status of the tissue. We propose a novel acquisition method that combines advantages of the current simultaneous and sequential acquisition. The proposed method consists of a DCE-MRI acquisition interrupted by DSC-MRI acquisition. A new method for processing of the DCE-MRI data is proposed which takes the interleaved acquisition into account. Analysis of both the DCE- and DSC-MRI data is reformulated so that they are approximated by the same pharmacokinetic model (constrained distributed capillary adiabatic tissue homogeneity model). This provides a straightforward evaluation of the methodology as some of the estimated DCE- and DSC-MRI perfusion parameters should be identical. Evaluation on synthetic data showed an acceptable precision and no apparent bias introduced by the interleaved character of the DCE-MRI acquisition. Intravascular perfusion parameters obtained from clinical glioma data showed a fairly high correlation of blood flow estimates from DCE- and DSC-MRI, however, an unknown scaling factor was still present mainly because of the tissue-specific $r_{2}^{*}$ relaxivity. The results show validity of the proposed acquisition method. They also indicate that simultaneous processing of both DCE- and DSC-MRI data with joint estimation of some perfusion parameters (included in both DCE- and DSC-MRI) might be possible to increase the reliability of the DCE- and DSC-MRI methods alone.
\end{abstract}

Keywords: Perfusion imaging, contrast agents, brain tumors, DCE-MRI, DSC-MRI.

\section{INTRODUCTION}

Dynamic contrast enhanced MRI (DCE-MRI) and dynamic susceptibility contrast MRI (DSC-MRI) make quantitative estimates of the tissue perfusion parameters possible. DCE-MRI is used especially in tumor diagnostics and treatment monitoring, and partly in cardiology (see [1, 2, 3]). In contrast, DSC-MRI is used largely for diagnosing cerebral ischemic illnesses (see $[4,5]$ ). DCE-MRI and DSC-MRI perfusion methods are based on image sequences acquired following administration of a contrast agent. For each voxel, a contrast agent concentration time sequence is extracted from the recorded image sequence and is subsequently approximated by a pharmacokinetic model. The model is parametrized by the sought perfusion parameters. Most perfusion-imaging studies are based either on DCE- or DSC-MRI. Their advantages and disadvantages, when used alone, are reviewed first.

DCE-MRI is based on $T_{1}$-weighted image sequences. In DCE-MRI, a fairly slow process of the parenchymal phase of the contrast agent distribution is captured. Hence, the typical recording time is about 5-15 minutes. DCE-MRI is used mainly as a tool to measure the permeability of the capillary wall. The pharmacokinetic models used in DCE-MRI are reviewed in $[6,7]$. The simplest and widely used pharmacokinetic model is the Kety/Tofts model [8], which makes it possible to estimate the $v_{e}, k_{e p}$, and $K^{\text {trans }}$ (see Table 1, EES is the extravascular extracellular space). In the extended Kety/Tofts model, also $v_{b}$ is estimated [9]. More complex (second gen- 
eration) models have been introduced to model also the intravascular contrast agent distribution phase. The best known of these models are the two compartment exchange model (2CXM) [10], the adiabatic approximation of the tissue homogeneity model (aaTH) [11], and the distributed capillary adiabatic tissue homogeneity model DCATH [12]. With these models, additional perfusion parameters can be estimated: $T_{c}$, $F_{b}$, and $P S$ (Table 1). However, these second generation models require higher temporal resolution and higher signal-tonoise ratio $(S N R)$ so as not to be ill-posed due to the higher number of the estimated parameters and the fast dynamics of the vascular distribution phase [13].

DSC-MRI (see reviews $[4,14]$ ) is based on $T_{2}$ - or $T_{2}^{*}$ weighted image sequences. It has been used mostly for brain studies where no contrast agent extravasation occurs due to the blood brain barrier (BBB). Hence, only the vascular phase of the contrast agent distribution is assumed. This requires only one to two minutes of recording [15]. Contrast agent concentration curves are extracted from $T_{2} / T_{2}^{*}$-weighted image sequences. The vascular perfusion parameters can be estimated by approximating the contrast agent concentration time sequences with a nonparametric pharmacokinetic model or with a pharmacokinetic model assuming, e.g., a compartment approximation [14]. The estimated perfusion parameters are the cerebral blood flow, $C B F$ ( $F_{b}$ in DCE-MRI), cerebral blood volume, $C B V$ ( $v_{b}$ in DCE-MRI), and the mean intravascular transit time, $M T T$ ( $T_{c}$ in DCE-MRI). This model, however, is not valid for leaky capillaries. For these cases, the application of a prebolus (a small dose of the contrast agent applied before the DSC-MRI acquisition) is recommended [16], which decreases the contrast agent concentration gradient between the vascular space and the EES and, hence, suppresses the contrast agent extravasation. Another effect making absolute quantification of $C B V$ and $C B F$ a challenge is the dependence of the DSC-MRI signal (namely $r_{2}^{*}$ and $r_{2}$ relaxivity) on the vessel size and structure [14].

It is advantageous to apply both DCE- and DSC-MRI together to exploit their complementary information about the permeability and blood flow of the tissue or to correct for unwanted $T_{1}$ and $T_{2}^{*}$ effects $[17,18,19]$. DCE- and DSCMRI data can be acquired simultaneously using multi gradient echo techniques. They can be combined with saturation recovery preparation $[20,21]$ or with spin echo and echo planar imaging (EPI) techniques [22]. By acquiring data for several (at least two) echo times, it is possible to estimate $T_{2}^{*}$ (and possibly also $T_{2}$ for the combined spin- and gradientecho acquisition) and $T_{1}$ separately. Consequently, DCE-MRI concentration time curves can be corrected for degradation caused by shortening of $T_{2}^{*}$ and the DSC-MRI concentration time curves can be corrected for impairment caused by shortening of $T_{1}$. Regardless of these corrections, in the case of contrast agent extravasation (e.g., in tumors), the DSCMRI contrast agent concentration as derived from $T_{2}^{*}$ or $T_{2}$ is affected by the time varying gradient of the contrast agent concentration between the intra- and extravascular space. A general disadvantage of simultaneous DCE- and DSC-MRI recording is that the acquisition of more gradient echoes and the need for a sufficient signal-to-noise ratio even for higher echo times leads to lower spatial and/or temporal resolution of the acquired images compared to standard separate DCE and DSC acquisitions.

Another possibility of acquiring both DCE- and DSC-MRI data is a sequential acquisition [23]. DCE-MRI is recorded first, because the contrast agent bolus is also a prebolus for the following DSC-MRI acquisition, suppressing contrast agent extravasation in the subsequent DSC-MRI. This makes it possible to use an intravascular pharmacokinetic model for the DSC-MRI analysis. Compared to the simultaneous DCEDSC method mentioned above, the sequential acquisition method is more time demanding, but it can take advantage of a higher spatial and/or temporal resolution. Complete suppression of contrast agent extravasation in DSC-MRI can be achieved by using a high molecular weight contrast agent (so far allowed only for preclinical experiments) [17].

In this paper, a three-segment DCE-DSC-DCE acquisition method is used. It combines the advantages of both the simultaneous and sequential techniques. By interrupting the DCE acquisition and inserting the DSC acquisition, the prebolus suppression of extravasation is kept as well as a short acquisition duration (corresponding to that of a DCE experiment). At the same time, spatial and/or temporal resolution higher than with the multi echo approach is achieved. Our preliminary versions of this acquisition method can be found in [24] and [25]. In this paper, the proposed method is evaluated on simulated data and on a set of clinical data.

Pharmacokinetic models that impose the same assumptions on both DCE- and DSC-MRI are used, based on the constrained DCATH pharmacokinetic model [26], with modifications accounting for the interrupted acquisition and the prebolus extravasation suppression. This provides a straightforward evaluation of the methodology as some of the estimated DCE- and DSC-MRI perfusion parameters (intravascular perfusion parameters: blood flow, blood volume, and mean transit time) should be identical. Such comparison can provide an insight into the possibility of a joint estimation of the intravascular perfusion parameters from DCE- and DSC-MRI.

\section{SubJects \& MeThods}

Assuming stationary perfusion parameters (i.e., timeinvariance during acquisition) and linearity of the modeled system, the contrast agent concentration time sequences extracted from the recorded DSC-MRI or DCE-MRI image sequence for each voxel or tissue region of interest (ROI) can be modeled using the pharmacokinetic model:

$$
C_{R O I}(n)=\frac{F_{p}}{1-H c t_{L V}}\left(C_{A I F} * I R F\right)(n),
$$

where $n$ is the time index, $(*)$ is the discrete convolution operator, $C_{A I F}(n)$ is the arterial input function (AIF), i.e. the contrast agent concentration in blood measured in an artery feeding the tissue ROI, $\mathrm{Hct}_{L V}$ is hematocrit in large vessels used for conversion of the contrast agent concentration in blood, $C_{A I F}(n)$, to blood plasma concentration, $C_{p}(n)$. The sequence 
$\operatorname{IRF}(n)$ is the impulse residue function (the probability that a contrast agent particle after an ideal instantaneous bolus injection is still present in the tissue ROI at the time index $n$ ). The perfusion parameters $\left(F_{p}\right.$ and the parameters of $\left.\operatorname{IRF}(n)\right)$ are estimated by deconvolution, formulated as least-squares fitting of the model (1) to the measured sequence, $C_{R O I}(n)$, assuming that $C_{A I F}(n)$ is known, e.g., measured, see below.

\subsection{Three-segment acquisition protocol}

The proposed interleaved acquisition protocol (Fig. 1, right column) is designed to include both contrast enhanced techniques in the shortest possible time window. First, the calibration DCE-MRI sequence is applied, which is used to convert signal intensity to contrast agent concentration, e.g., the multi flip angle acquisition (multiple $F A$ ) [27]. This sequence is followed by administration of the contrast agent and the first part of the contrast DCE-MRI acquisition. This part captures the arterial phase and the start of the parenchymal phase of the contrast agent distribution. Then, high resolution anatomical scans are acquired, followed by the second bolus of the contrast agent and the DSC-MRI acquisition. Finally, the second part of the contrast DCE-MRI sequence is recorded (no contrast agent applied). This part captures the parenchymal phase of the contrast agent distribution.

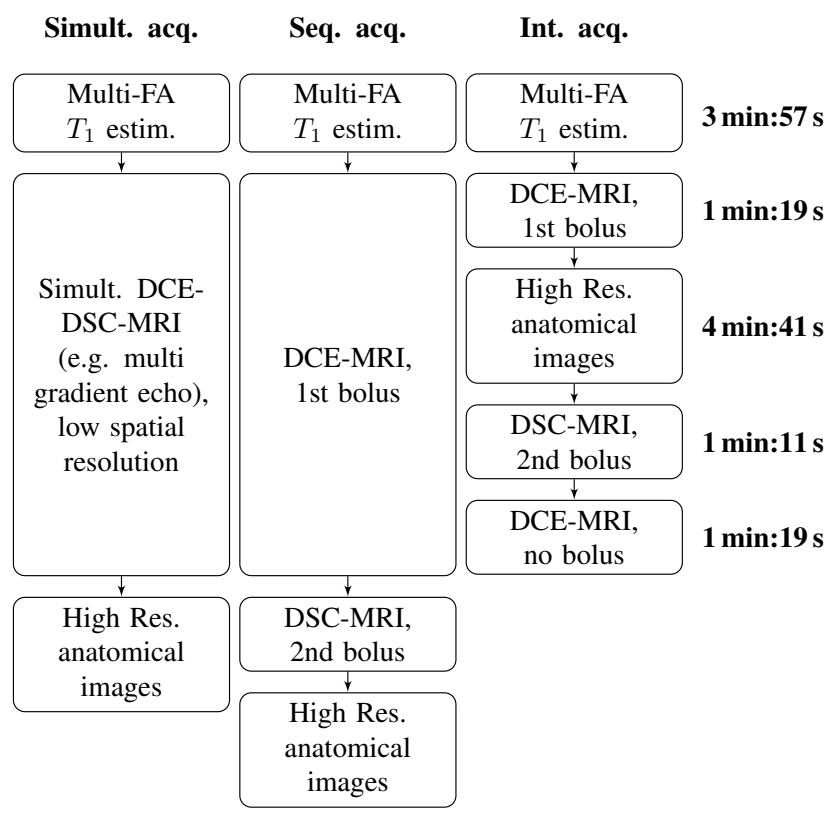

Fig. 1. Schematic comparison of the proposed Interleaved acquisition (right column), the simultaneous DCE- DSC-MRI acquisition (left), and the sequential DCE- DSC-MRI acquisition (center column). Total duration of the Interleaved protocol is $12 \mathrm{~min}: 27 \mathrm{~s}$.

\subsection{Arterial input functions}

For processing of the DCE-MRI recording, the two segments of the AIF are derived from the two DCE-MRI contrast recordings. They are calculated as the average contrast agent concentration sequence within a manually drawn region in a feeding artery. To provide the missing part of the AIF corresponding to the missing data between the two DCE-MRI contrast recordings, the measured AIF segments are approximated by a parametric model. It is derived from Parker's AIF model [28]:

$$
\begin{aligned}
& C_{p 1}(n, \mathbf{r})=\sum_{i=1}^{2} \frac{A_{i}}{\sigma_{i} \sqrt{2 \pi}} \exp \left(\frac{-\left(n-N_{i}\right)^{2}}{2 \sigma_{i}^{2}}\right)+ \\
&+\frac{\alpha \exp (-\beta n)}{1+\exp (-s(n-\tau))}
\end{aligned}
$$

where $\mathbf{r}=\left[A_{1}, A_{2}, N_{1}, N_{2}, \sigma_{1}, \sigma_{2}, \alpha, \beta, s, \tau\right]$ is the vector of Parker's AIF parameters. To account for the application of two boluses, the sum of two delayed and scaled Parker's AIFs is used:

$$
C_{p}(n, \mathbf{r})=C_{p 1}(n, \mathbf{r})+D_{R} C_{p 1}\left(n-N_{s}, \mathbf{r}\right) .
$$

The parameter $N_{s}$ is the time delay between the applications of the two boluses, estimated as the delay between the peaks in the measured AIF signals of the first-phase DCE-MRI and the DSC-MRI recordings. The parameter $D_{R}$ is the ratio of the DSC-MRI and the DCE-MRI bolus doses. An example of the measured AIF segments and the fitted AIF model (3) is given in Fig. 2.
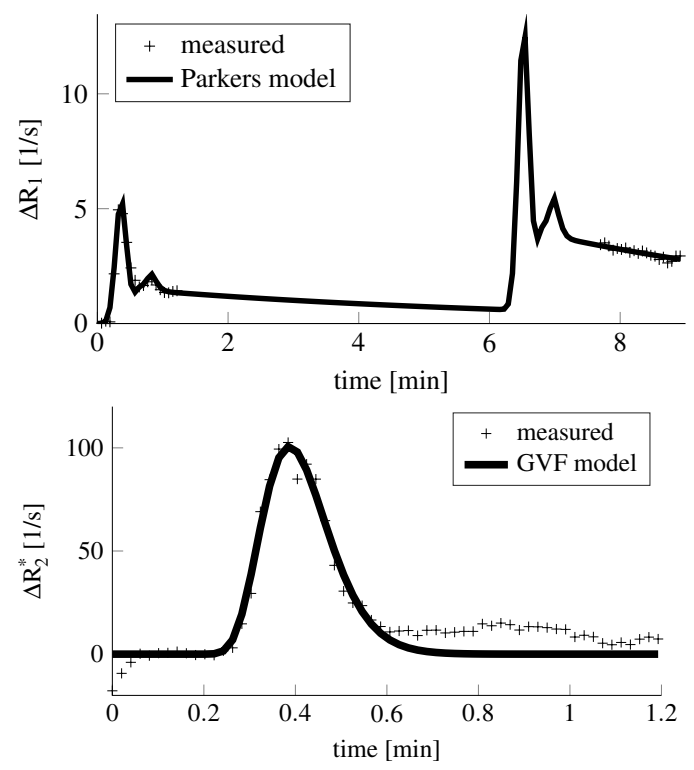

Fig. 2. Approximation of the DCE-MRI AIF sequences by Parker's model (the second contrast agent bolus had a two-fold higher dose than the first bolus) (upper). Approximation of the DSC-MRI AIF sequence by the GVF model (lower).

To minimize the partial volume effect, the individual examination-specific AIFs are scaled to have the same area under the curve (AUC) as the AIF with the maximum AUC in the data set, as in [29]. The same contrast agent dose in all examinations is assumed. The AUC is calculated numerically from the AIF model (3) in the time interval from time zero to the point of almost complete tracer elimination. The elimination time is determined as the time when the AIF level reaches a defined negligible fraction of its maximum (here 1/250). 
Table 1. Perfusion parameters of the aaTH model

\begin{tabular}{|c|c|c|}
\hline symbol & description & unit \\
\hline$\overline{F_{p}}$ & $\begin{array}{l}\text { Blood plasma flow per unit } \\
\text { volume of tissue }\end{array}$ & $\overline{\mathrm{mL} / \mathrm{min} / 100 \mathrm{~mL}}$ \\
\hline$F_{b}$ & $\begin{array}{l}\text { Whole blood flow per unit } \\
\text { volume of tissue }\end{array}$ & $\mathrm{mL} / \mathrm{min} / 100 \mathrm{~mL}$ \\
\hline$v_{p}$ & $\begin{array}{l}\text { Blood plasma volume per } \\
\text { unit volume of tissue }\end{array}$ & $\mathrm{mL} / 100 \mathrm{~mL}$ \\
\hline$v_{b}$ & $\begin{array}{l}\text { Whole blood volume per } \\
\text { unit volume of tissue }\end{array}$ & $\mathrm{mL} / 100 \mathrm{~mL}$ \\
\hline$E$ & Initial extraction ratio & - \\
\hline$K^{\text {trans }}$ & $\begin{array}{l}\text { Volume transfer constant be- } \\
\text { tween blood plasma and } \\
\text { EES }\end{array}$ & $1 / \min$ \\
\hline$k_{e p}$ & $\begin{array}{l}\text { Rate constant between EES } \\
\text { and blood plasma }\end{array}$ & $1 / \mathrm{min}$ \\
\hline$T_{c}$ & Mean capillary transit time & $\mathrm{s}$ \\
\hline$v_{e}$ & $\begin{array}{l}\text { Volume of the EES per unit } \\
\text { volume of tissue }\end{array}$ & $\mathrm{mL} / 100 \mathrm{~mL}$ \\
\hline$P S$ & $\begin{array}{l}\text { Permeability surface area } \\
\text { product per unit volume } \\
\text { of tissue }\end{array}$ & $\mathrm{mL} / \mathrm{min} / 100 \mathrm{~mL}$ \\
\hline$B A T$ & Bolus arrival time & $\mathrm{s}$ \\
\hline
\end{tabular}

In the case of DSC-MRI AIF selection, a semiautomatic searching algorithm based on [30] is used. As in DCE-MRI, the individual examination specific AIFs are scaled to the maximum AUC in the DSC-MRI data set, to minimize the partial volume effect. In contrast to DCE-MRI, the AUC is calculated only from the first pass of the contrast agent, i.e. recirculation effects (secondary peaks) and contrast agent extravasation are not taken into account. For this purpose, a special form of the gamma variate function (GVF) with decoupled model parameters [31] is used to approximate the first pass part of the DSC-MRI AIF (here $2 / 3$ of the samples of the whole signal). The first pass AUC part is then determined as the AUC of the complete GVF function, see Fig. 2 for an example.

\subsection{Impulse residue functions}

For DCE-MRI, $\operatorname{IRF}(n)$ is modeled by the DCATH model with a truncated normal distribution of the intravascular transit times, which does not allow negative transit times [12, 13]:

$$
\begin{aligned}
& \operatorname{IRF}(n)=\operatorname{IRF}_{v}(n)+\operatorname{IRF}_{p}(n) \\
& \operatorname{IRF}_{v}(n)=1-\frac{1}{2 N_{f}}\left[\operatorname{erf}\left(\frac{n T_{s}-T_{c}}{\sqrt{2} \sigma}\right)+\operatorname{erf}\left(\frac{T_{c}}{\sqrt{2} \sigma}\right)\right] \\
& \operatorname{IRF}_{p}(n)=\frac{E}{2 N_{f}} \exp \left[\frac{k_{e p}{ }^{2} \sigma^{2}}{2}+k_{e p}\left(T_{c}-n T_{s}\right)\right] \times \\
& \times\left\{\operatorname{erf}\left[\frac{\left(n T_{s}-T_{c}\right)}{\sqrt{2} \sigma}-\frac{k_{e p} \sigma}{\sqrt{2}}\right]+\operatorname{erf}\left[\frac{T_{c}}{\sqrt{2} \sigma}+\frac{k_{e p} \sigma}{\sqrt{2}}\right]\right\},
\end{aligned}
$$

where the error function $\operatorname{erf}$ is defined as

$$
\operatorname{erf}(n)=\frac{2}{\sqrt{\pi}} \int_{0}^{n} \mathrm{~d} x \exp \left(-x^{2}\right)
$$

and $N_{f}$ is a normalization factor accounting for truncation of the normal distribution of the intravascular transit times [12]:

$$
N_{f}=\frac{1}{2}\left[1+\operatorname{erf}\left(\frac{T_{c}}{\sqrt{2} \sigma}\right)\right] .
$$

To decrease the number of free parameters, the DCATH model was modified by fixing the variation of the intravascular transit time, $\sigma$, to a small value (as proposed in [26]). Hence, the modified DCATH model is an approximation to the aaTH model still with the advantage of a continuous formulation of the mean intravascular transit time, $T_{c}$ [13].

For small $\sigma$, the mean value of the normal intravasculartransit-time distribution before truncation is used as a good estimate of the mean intravascular transit time, $T_{c}$, corresponding to the truncated normal distribution [12].

The quantities $E, k_{e p}$, and $T_{c}$ are the tissue specific perfusion parameters to be estimated, along with $F_{p}$ (Table 1$)$. The remaining perfusion parameters can be derived from these parameters as: $v_{e}=E F_{p} / k_{e p}$ and $v_{p}=F_{p} T_{c}$.

An additional parameter to be estimated is the bolus arrival time, $B A T$, i.e. the delay between the rising edge of the AIF, $C_{A I F}(n)$, and of the tissue contrast agent concentration time sequence, $C_{R O I}(n)$. Here, it is estimated according to [13] in the Fourier domain, where it has a continuous formulation.

For DSC-MRI, the model in (4) is modified by assuming no extravasation, i.e. $E=0$. Hence, only the vascular part, $I R F_{v}(n)$, is used. This formulation approximates the standard box IRF (used in DSC-MRI, e.g. [32]) to allow a continuous formulation of $T_{c}$. The estimated perfusion parameters are $F_{p}, T_{c}$, and $B A T$. Blood plasma volume is then derived as $v_{p}=F_{p} T_{c}$ as for DCE-MRI.

\subsection{Synthetic data, Monte Carlo simulations}

For validation of the proposed interleaved DCE-MRI method, it was compared to the standard DCE-MRI method using synthetic data. Different tissue types and $S N R$ values $(5,6,7,8$, $9,10,15,20,25,30,35,40,50$, and 60 ) were simulated. The effect of low temporal sampling rate of DCE-MRI (as used here for the clinical data acquisition, see below) was also simulated and tested.

The arterial concentration time curve for the AIF extraction was generated using (3) and the parameters from the original paper [28]. Three different IRFs (parametrized with $\mathbf{p}_{\mathbf{t}}=\left\{F_{p}, E, v_{e}, T_{c}, B A T\right\}$ ) were generated, simulating three tissue types. The first tissue represented healthy prostate $\left(\mathbf{p}_{\mathbf{t 1}}=\{0.21,0.65,0.35,18.60,3.94\}\right.$ [33] $)$, the second tissue was a prostate tumor $\left(\mathbf{p}_{\mathbf{t} 2}=\{0.08,0.80,0.21,60.00,3.94\}\right.$ [33]), and the third tissue was a brain glioma tumor $\left(\mathbf{p}_{\mathbf{t} 3}=\right.$ $\{0.05,0.16,0.08,12.00,3.94\}$ [34]). The units of the perfusion parameters were according to Table 1 . The BAT parameters were set equally to $3.94 \mathrm{~s}$ for all three tissues. 
Noise-free high temporal resolution concentration time curves $C_{R O I}(n, \mathbf{p})$ were generated by a discrete convolution of the AIF and $\operatorname{IRF}(n, \mathbf{p})$. Four acquisition and processing types were evaluated: the standard and the interleaved DCE method, each with a high and low temporal resolution, $T_{s}=0.99 \mathrm{~s}$ and $T_{s}=3.94 \mathrm{~s}$ (same as for the clinical data, see below), respectively. In case of low temporal resolution, the concentration time curves were undersampled by a factor of 4. Then, zero mean Gaussian noise was added. The standard deviation of noise, $\sigma_{\text {noise }}$, was calculated according to the chosen $S N R$ level, defined as

$$
S N R=\frac{1}{N \sigma_{\text {noise }}} \sum_{n=1}^{N} C_{R O I}(n, \mathbf{p}) .
$$

To simulate noise of the measured arterial concentration time curve (for AIF extraction), the same value of $\sigma_{\text {noise }}$ was used for noise added to the arterial curve. For each level of the $S N R$ and each tissue type, 500 noise realizations were generated.

Finally, the standard (complete) and interleaved (segment of $C_{R O I}(n, \mathbf{p})$ of the same position and duration was left out as in clinical recordings, see below) data sets were processed using the standard perfusion analysis and the method proposed for the interleaved acquisition, respectively.

A relative estimation error of each perfusion parameter $x$ was calculated as

$$
\delta_{x}=\frac{|\widehat{x}(w)-x|}{x} 100,
$$

where $w$ is the index of noise realization, $\widehat{x}$ is the estimated value of the perfusion parameter and $x$ is its ground-truth value. To study the effect of the missing segment in the interleaved acquisition and of low sampling rate, a median relative error was calculated for each $S N R$ from $\delta_{x}$ of all intravascular parameters $\left(F_{p}, v_{p}, T_{c}, B A T\right)$.

\subsection{Clinical data}

The comparison of intravascular perfusion parameters was tested on data sets from 6 patients: 3 patients (age 51, 58, and 68) with one or more brain glioma metastases and 3 patients with primary glioma (age 70, 72, and 72). All involved patients were scanned upon ethical approval and a written informed consent.

Data acquisition was done using the MRI scanner Achieva 1.5 T (Philips Healthcare, Eindhoven, Netherlands) with a 16 channels receive head coil SENSE-NV-16. The contrast agent Gadobutrol (Gadovist, Bayer Pharma AG, Berlin, Germany) was applied at the dose $5 \mathrm{~mL}$ for DCE-MRI and $10 \mathrm{~mL}$ for DSC-MRI, i.e. the bolus dose ratio was $D_{R}=2$, application speed $5 \mathrm{~mL} / \mathrm{s}$, pressure limit $325 \mathrm{lb} / \mathrm{in}^{2}$ (psi). Both applications were followed by the same amount of $20 \mathrm{~mL}$ saline at the same speed $5 \mathrm{~mL} / \mathrm{s}$. The contrast agent was administered to the left arm vein using the power injector (Spectris Solaris EP, Medrad, Warrendale, USA) to provide a consistent and reproducible administration speed and dosing.

\section{DCE-MRI acquisition and processing}

For the DCE-MRI calibration recording, the 3D Fast Low Angle Shot (FLASH) sequence was used, with the parameters $\mathrm{TE} / \mathrm{TR}=1.80 / 3.83 \mathrm{~ms}$, temporal resolution $T_{s}=3.94 \mathrm{~s}$, flip angles $\left(\mathrm{FA}=\left\{10,20,30^{\circ}\right\}\right), 20$ slices per volume, acquisition matrix $64 \times 64$, and acquisition duration of $1 \mathrm{~min} 19 \mathrm{~s}$. It was followed by administration of a contrast agent bolus and the DCE-MRI contrast acquisition using the same parameters as for the DCE-MRI calibration recording with flip angle $30^{\circ}$, 20 cycles (time samples), and acquisition duration of $1 \mathrm{~min}$ $19 \mathrm{~s}$. Then, the DSC-MRI sequence and acquisition of high resolution anatomical images were performed (see below for acquisition details). This acquisition phase took 5 min $52 \mathrm{~s}$. Subsequently, the DCE-MRI postcontrast sequence was acquired using the same acquisition parameters as for the first DCE-MRI contrast acquisition (no bolus). The complete interleaved acquisition protocol took $12 \mathrm{~min} 27 \mathrm{~s}$.

In the preprocessing phase, the DCE-MRI contrast recordings were converted from signal intensity to the change of the longitudinal relaxation rate, $\Delta R_{1}$, using the method in [27]. The quantity $\Delta R_{1}$ was proportional to the concentration of the contrast agent.

The deconvolution algorithm was implemented as a constrained optimization (function lsqnonlin, MATLAB Optimization Toolbox, The MathWorks Inc., Natick, Massachusetts, U.S.A.). The standard deviation of the intravascular transit time, $\sigma$, in the DCATH IRF model was set to $T_{S} / 2$. To minimize the problem of optimization being trapped in a local optimum, the deconvolution algorithm was run from four different starting points (with different initial estimates of $T_{c}$ and $E$ ). The best solution was obtained as the one with the lowest final value of the criterion function.

\section{DSC-MRI acquisition and processing}

The DSC-MRI data were recorded using the 3D sequence PRinciples of Echo Shifting using a Train of Observations (sPRESTO) $[35,36]$ with the parameters $\mathrm{TE}_{0}=22.64 \mathrm{~ms}$, $\mathrm{TR}=15.43 \mathrm{~ms}, \mathrm{FA}=7^{\circ}$, one $T R$ interval echo shift $n_{s}=1$ and temporal resolution $1.19 \mathrm{~s}, 30$ slices, acquisition matrix $64 \times 64$ voxels, 60 cycles, acquisition duration $1 \mathrm{~min} 11 \mathrm{~s}$. Signal intensity of the acquired images was converted to the transversal relaxation rate change, $\Delta R_{2}^{*}$, using the standard mono-exponential relationship ([37]), with $T E=T E_{0}+n_{s} T R$ [35]. Then, the time sequences $\Delta R_{2}^{*}$ were converted to the contrast agent concentration sequences by dividing by $r_{2}^{*}$. For the tissue voxels, $r_{2 \text { tissue }}^{*}=44 \mathrm{mM}^{-1} \mathrm{~s}^{-1}$ and for the AIF, $r_{\text {2blood }}^{*}=7.6 \mathrm{mM}^{-1} \mathrm{~s}^{-1}[38]$. This is a standard approach for absolute quantification in DSC-MRI (the values in [38] were given for Gadopentetate dimeglumine; the same ratio of $r_{2 \text { blood }}^{*} / r_{2 \text { tissue }}^{*}$ was assumed also for Gadobutrol contrast agent used in our study, because $r_{2}^{*}$ of glioma tissue was not given in literature, to the best of our knowledge). The deconvolution algorithm was implemented in the same way as for the DCE-MRI part. 


\section{Comparison of perfusion parameters}

To evaluate the proposed DCE- and DSC-MRI acquisition and analysis methods on clinical data, the intravascular perfusion parameters estimated by the DCE- and DSC-MRI analyses were compared. Blood flow and volume were derived from plasma flow and volume using literature based values for the hematocrit in small vessels, $H c t_{S V}=0.28$, and large vessels, $H c t_{L V}=0.40$ [39]. Denoting their ratio as $r_{H c t}=H c t_{S V} / H c t_{L V}$, blood flow was calculated as $F_{b}=F_{p} /\left(1-r_{H c t} H c t_{L V}\right)$. Using the central volume theorem, blood volume was calculated as $v_{b}=F_{b} T_{c}$.

To image the same brain volume using both DCE- and DSC-MRI and to provide a good temporal and spatial resolution, DCE- and DSC-MRI data were recorded with different geometries. The DSC-MRI acquisition method allowed for a high temporal (1.19s) and spatial (30 slices) resolution, while for the DCE-MRI acquisition method lower temporal and spatial resolution was achievable (3.83 s, 20 slices). Hence, the DCE-MRI and DSC-MRI results were evaluated by comparing the statistical distributions of the perfusion parameter values estimated within the tumor regions.

The perfusion parameter maps were segmented to obtain the tumor regions as follows. A binary 3D mask of the tumor region was obtained from high-resolution $T_{1}$ - and $T_{2}$-weighted images and their blurred versions by applying a Support Vector Machine (SVM) classification algorithm [40]. It is based on the search for an optimum hyperplane dividing the data into two groups with a maximum distance between the hyperplane and the data in both groups closest to the hyperplane. To allow a nonlinear formulation of the dividing hyperplane, a Gaussian radial basis function was used as a kernel of the SVM algorithm, mapping the input feature space into a higher dimension feature space [40].

To train the segmentation method for the given examination data set, voxels inside and outside the tumor were manually selected. The intensity values at the indicated points in both $T_{1}$ - and $T_{2}$-weighted images were used as an input for training of the segmentation model algorithm.

The resulting binary 3D masks of the tumor were then geometrically transformed and resampled to the sampling grid of the perfusion images. The perfusion parameters within the tumor mask were extracted and compared using scatter plots with denoted medians and the 25 th and 75 th percentiles. In DCE-MRI, the voxels with noisy signals $(S N R<5)$ were omitted (estimation of $S N R$ for clinical data is described below).

The high resolution $T_{1}$-weighted images were acquired using a 3D FLASH sequence with spatial resolution $0.8 \times$ $0.8 \times 1.6 \mathrm{~mm}$, acquisition matrix $300 \times 298$ voxels, 209 slices, $\mathrm{TE}=4 \mathrm{~ms}$, TR $=25 \mathrm{~ms}$, and flip angle $30^{\circ}$. The high resolution $T_{2}$-weighted images were acquired using a turbo spin echo sequence with spatial resolution $0.45 \times 0.45 \times 5.00 \mathrm{~mm}$, acquisition matrix $384 \times 299$ voxels, 22 slices, $\mathrm{TE}=100 \mathrm{~ms}$, $\mathrm{TR}=4400 \mathrm{~ms}$, and flip angle $90^{\circ}$.

To relate the $S N R$ of the simulated data to the clinical data, median $S N R$ within the tumor regions of all patients was calculated from all tumor voxels according to (7). Standard de- viation of the noise $\left(\sigma_{\text {noise }}\right)$ was estimated as follows. The postcontrast segment (second DCE acquisition) was approximated by a monoexponential function. This function was then subtracted from the measured signal. Finally, $\sigma_{\text {noise }}$ was estimated from the residuals. Mean signal value in (7) was calculated as a mean of the measured signal.

\section{RESUlts}

\subsection{Synthetic DCE-MRI data}

Fig. 3 shows the results of the Monte Carlo analysis. The interleaved method led to increased perfusion parameter estimation error compared to the standard DCE method. Also, lower temporal resolution led to higher estimation errors. The estimation error decreased with increasing $S N R$. The median $S N R$ estimated for the clinical DCE data was 9.7. This relates the clinical data to the simulated data set with $S N R=10$. For this case $(S N R=10)$, the estimation error of the intravascular perfusion parameters was below $31 \%$ (Fig. 3).

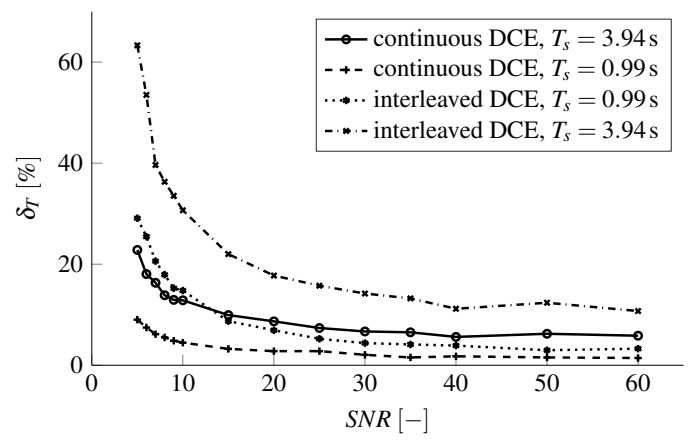

Fig.3. Results of the Monte Carlo simulation, median relative errors estimated from intravascular parameters $\left(F_{b}, v_{b}, T_{c}, B A T\right)$ as a function of the $S N R$

The boxplots in Fig. 4 show detailed results for $S N R=10$ and the brain tumor tissue as the case closest to the used clinical data set. The boxplots show the same trends as Fig. 3 . Importantly, these plots indicate that the omitted segment in the interleaved DCE acquisition did not induce any additional systematic bias of the perfusion parameter estimates (boxplots B), while low temporal resolution did for $T_{c}$ and $v_{b}$ (boxplots $\mathrm{A}$ and $\mathrm{C}$ ).

\subsection{Clinical DSC- and DCE-MRI data}

Fig. 2 shows examples of the measured and approximated AIFs for DCE-MRI and DSC-MRI. Fig. 5 shows examples of approximation of the tissue contrast agent concentration sequences by the convolutional model (1) and the estimated IRF.

Figure 6 (left) shows a result of the tumor region segmentation. The resulting perfusion-parameter maps (Fig. 6 (top and bottom row)) done in approximately the same slice position showed similar structures in the parametric maps of $F_{b}$ and $v_{b}$, however, they were differently scaled. The $T_{c}$ maps were clearly different. 

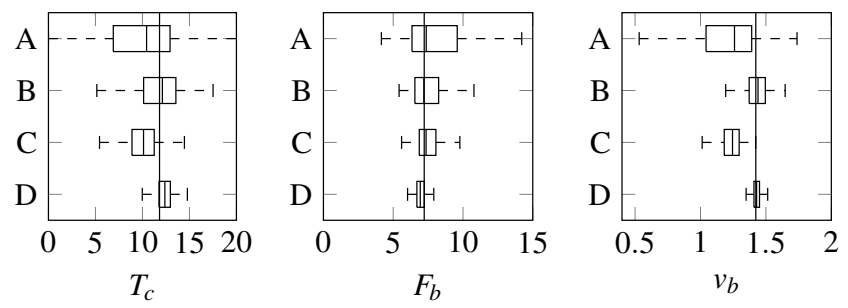

Fig.4. Synthetic data, $S N R=10$, brain tumor tissue, boxplots comparing different DCE-MRI techniques, A: interleaved DCE with $T_{s}=3.94 \mathrm{~s}$, B: interleaved DCE with $T_{s}=0.99 \mathrm{~s}, \mathrm{C}$ : continuous DCE with $T_{s}=3.94 \mathrm{~s}$ and D: continuous DCE with $T_{s}=0.99 \mathrm{~s}$. Solid vertical lines denote the ground truths. The units are $v_{b}[\mathrm{~mL} / 100 \mathrm{~mL}]$, $F_{b}[\mathrm{~mL} / \mathrm{min} 100 \mathrm{~mL}]$ and $T_{c}[\mathrm{~s}]$.
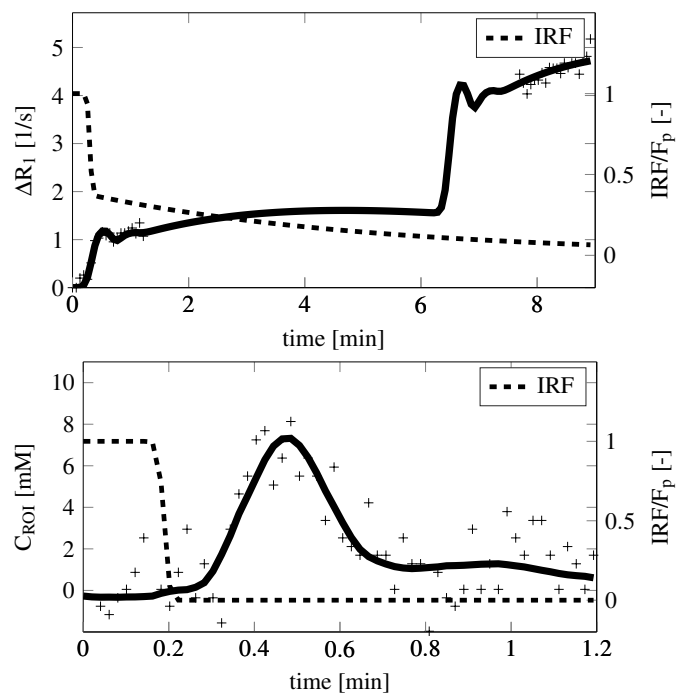

Fig.5. Approximation of the tissue contrast agent concentration sequence by the convolutional model for DCE-MRI (upper) and for DSC-MRI (lower).

The results of the complete data set are summarized as scatter plots (Fig. 7). In each scatter plot, the central mark is the median, the black solid lines denote the 25 th and 75 th percentiles, red lines represent linear regression of medians and black dashed lines are lines of unity slope. The estimated perfusion parameter values were in the expected range ac-
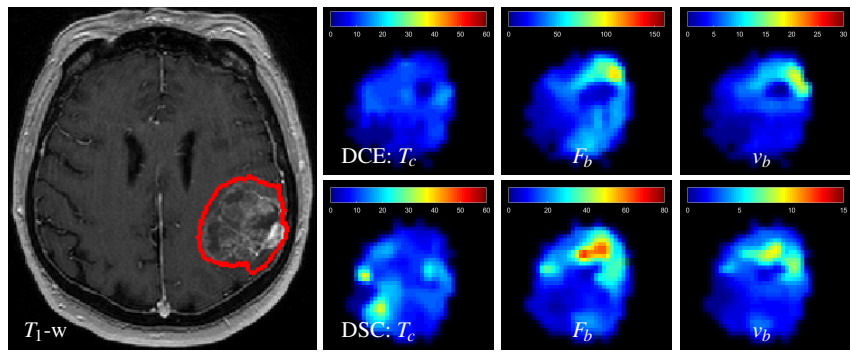

Fig.6. Example of tumor-region segmentation at high resolution $T_{1}$ weighted image /left/ and comparison of the DCE (top row) and DSC-MRI (bottom row) maps of $T_{c}[\mathrm{~s}], F_{b}[\mathrm{~mL} / \mathrm{min} / 100 \mathrm{~mL}]$ and $v_{b}[\mathrm{~mL} / 100 \mathrm{~mL}]$. cording to literature for gliomas ([34, 41, 42]). For each intravascular perfusion parameter, regression analysis was applied to the median values. The regression coefficient representing the slope of the regression line was 0.87 for $T_{c}, 0.32$ for $F_{b}$, and 0.14 for $v_{b}$. Hence, the perfusion parameter $T_{c}$ gave the best absolute agreement between the DCE-MRI and DSC-MRI methods. This is expected, because this parameter is not affected by scaling of the AIFs and by the vessel size dependency of $r_{2}^{*}$, in contrast to $F_{b}$ and $v_{b}$. The Pearson's median correlation coefficients between DCE-MRI and DSC-MRI medians were $R\left(T_{c}\right)=0.66, R\left(F_{b}\right)=0.84$, and $R\left(v_{b}\right)=0.55$. The observed correlation coefficients corresponded well with the level of bias in perfusion parameter estimates caused by low temporal resolution in simulated DCEMRI data (Fig. 4, boxplots A and C), i.e. no observed bias in $F_{b}$ estimates corresponded to the highest correlation coefficient of this perfusion parameter and the highest level of bias in $v_{b}$ corresponded to the lowest correlation coefficient of this perfusion parameter.
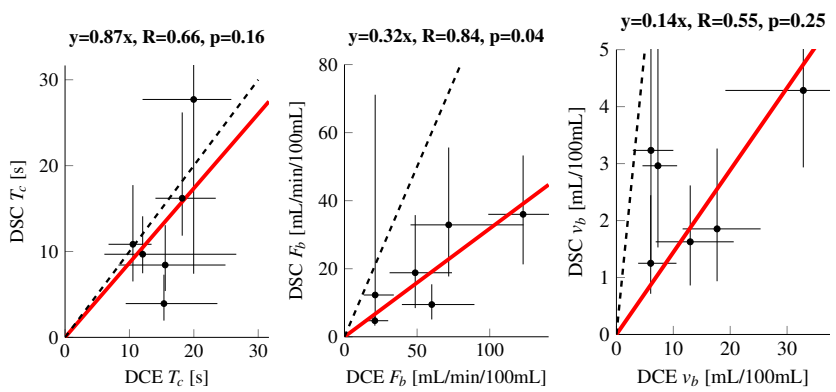

Fig. 7. Perfusion parameters scatter plots of patient data. In each scatter plot, the central mark is the median, the black solid lines denote the 25th and 75th percentiles, red lines represent linear regression of medians and black dashed lines are lines of unity slope.

\section{Discussion}

The proposed modifications of the DCE method were compared to the standard DCE method on synthetic data. The missing segment in the interleaved-acquisition DCE data led to a slightly decreased precision of perfusion parameter estimation and no bias (Fig. 4). Low temporal resolution $\left(T_{s}=3.94 s\right)$ had a similar effect of decreased precision, but in addition, it led to a bias in estimates of $T_{c}$ and $v_{b}$ (no bias was observed for $F_{b}$ ), see Fig. 4 . This suggests that among intravascular perfusion parameters, $F_{b}$ is the least sensitive to decreased temporal resolution in the sense of accuracy.

As expected, the combination of both lower temporal resolution and interleaved acquisition led to further decrease of precision (Fig. 4). The decrease in precision due to the proposed interleaved acquisition and lower temporal resolution clearly reflects fewer sampling points available for the perfusion analysis. This is certainly a limitation of the proposed interleaved method. In terms of median relative error of intravascular perfusion parameters, the combination of lower temporal resolution and interleaved acquisition had the highest error (approx. 30\%), compared to less than $20 \%$ error 
MEASUREMENT SCIENCE REVIEW, 18, (2018), No. 6, 262-271

obtained for other combinations of low/high temporal resolution and continuous/interleaved acquisition.

Testing of the complete DCE-DSC-DCE acquisition and processing scheme on glioma patient data sets showed that the estimated perfusion parameters were in the expected range, according to literature. The absolute match between the DCEand DSC-MRI perfusion parameters (tested using the slope of the regression line in scatter plots) showed a good agreement of $T_{c}$. On the other hand, the absolute match between the DCE- and DSC-MRI estimates of $F_{b}$ and $v_{b}$ was poor. This reflects an unknown scaling factor in DSC-MRI due to the tissue dependency of $r_{2}^{*}$ which is still the main problem in absolute quantification of $F_{b}$ and $v_{b}$ in DSC-MRI.

The correlation coefficients calculated from the clinical data results were in line with the accuracy of the modified DCE-MRI method resulting from the evaluation on synthetic data (done for the same SNR and tissue type as used in the clinical recordings). Hence, a fairly high correlation coefficient was obtained for $F_{b}$ because its estimates had no bias on synthetic data. Correspondingly, the lowest correlation coefficient was obtained for $v_{b}$ because it demonstrated the strongest bias on synthetic data.

As was shown on synthetic data, the main source of bias was low temporal resolution due to $3 \mathrm{D}$ mode of DCE sequence. A higher sampling rate can be reached with $2 \mathrm{D}$ acquisition. However, 2D acquisitions lack a higher volume coverage. The temporal resolution was sufficient for the tissue concentration function because it can be treated as a low pass filtered version of the IRF (the low-pass filter is the AIF). The low temporal resolution is, however, insufficient for sampling of the AIF. Hence, the main source of errors was probably the AIF estimation. The problem of AIF measurement could be solved by AIF estimation based on blind deconvolution [26], where the AIF is estimated from one or several tissue contrast agent concentration sequences. These sequences can be sampled with a lower temporal resolution because they develop more slowly compared to the arterial signal, as explained above and shown in [43]. The AIF estimation scheme based on the measured arterial signal as used here was selected as the first choice, because it has a wider acceptance than the blind deconvolution estimation.

Another approach to the low temporal sampling rate could be the use of fixed population based AIF parameters, as presented in [28]. This would, however, ignore interpatient differences.

Another source of inaccuracy is the assumption of no extravasation of the contrast agent in processing of the DSCMRI data. Even though it was suppressed by the pre bolus applied during the first DCE-MRI acquisition step, the remaining contrast agent exchange between the vascular and extravascular spaces was not taken into account. Also, the $r_{2}^{*}$ relaxivities of tissue and blood were fixed to literature values. However, $r_{2}^{*}$ has been shown to be tissue specific (see $[38,37])$. This is a general problem for absolute quantification in DSC-MRI. This problem could be solved by formulation of $r_{2}^{*}$ as an additional "perfusion" parameter to be estimated. The theory and application of this approach to simul- taneously acquired DCE-DSC data was described in [18, 21]. However, it could be applied to the interleaved DCE-DSCDCE acquisition in a similar way.

The advantage of the proposed interleaved acquisition method over the standard sequential DCE-DSC protocol is time efficiency. The acquisition length of the presented protocol is identical with the acquisition length required for a standard DCE-MRI recording. On the other hand, the missing part of DCE in the interleaved method deteriorates the precision of the perfusion parameter estimates.

Compared to the simultaneous acquisition of DCE- and DSC-MRI data based on multi echo sequences, the interleaved approach improves spatial and/or temporal resolution. This is because no additional time is needed for acquisition of additional echoes. Again, this is at the cost of lower precision of the perfusion parameter estimates. Another advantage of the interleaved acquisition, compared to the multi echo sequences, is its inherent dual bolus nature providing suppression of contrast agent extravasation. However, the dual bolus feature could be incorporated also to the multi echo acquisition by applying a second contrast agent bolus during the acquisition.

\section{CONCLUSIONS}

We have proposed a new acquisition and processing method for measurement of DCE- and DSC-MRI which combines advantages of the current sequential and simultaneous acquisition techniques. We have applied the same pharmacokinetic model in processing of the DCE- and DSC-MRI data. This makes it possible to compare, in absolute terms, some of the perfusion parameters estimated from the DCEand DSC-MRI, namely the intravascular perfusion parameters: vascular mean transit time, blood flow, and volume. The evaluation on clinical data showed a fairly high correlation of blood flow estimates from DCE- and DSC-MRI. However, an unknown scaling factor was still present, mainly due to the tissue-specific character of $r_{2}^{*}$.

Thanks to the unified pharmacokinetic modeling od DCEand DSC-MRI data, this work can be considered an initial study for a possible joint estimation of the intravascular perfusion parameters. Such joint estimation can be formulated as a simultaneous approximation of the voxel based DCEand DSC-MRI contrast agent concentration sequences by a pharmacokinetic model where the intravascular parameters are the same for the DCE and DSC parts. This would decrease the number of parameters to be estimated compared to independent DCE- and DSC-MRI analysis. It should lead to improved estimation accuracy. To perform this step, the limitations of this study (mainly low temporal resolution of DCE-MRI, inaccurate AIF, remaining extravasation of the contrast agent in DSC-MRI, and tissue dependent $r_{2}^{*}$ ) have to be solved.

\section{ACKNOWLEDGEMENT}

The research was supported by the Czech Science Foundation (project GA16-13830S), by the Min- 
istry of Education, Youth and Sports of the Czech Republic (MEYS projects LO1212, CZ.1.05/2.1.00/01.0017, and CZ.02.1.01/0.0/0.0/16_013/0001775), and by the Czech Academy of Sciences (project RVO:68081731). Further support was provided by the National Sustainability Programme (project LO1401) and by the SIX Center and CzechBioImaging (MEYS LM2015062) infrastructures.

\section{REFERENCES}

[1] Jerosch-Herold, M. (2010). Quantification of myocardial perfusion by cardiovascular magnetic resonance. Journal of Cardiovascular Magnetic Resonance, 12(1), 57.

[2] Jackson, A., Buckley, D.L., Parker, G.J.M. (eds.) (2004). Dynamic Contrast-Enhanced Magnetic Resonance Imaging in Oncology (Medical Radiology). Springer.

[3] Tofts, P. (ed.) (2004). Quantitative MRI of the Brain: Measuring Changes Caused by Disease. Wiley.

[4] Willats, L., Calamante, F. (2013). The 39 steps: evading error and deciphering the secrets for accurate dynamic susceptibility contrast MRI. NMR in Biomedicine, 26(8), 913-931.

[5] Calamante, F., Gadian, D., Connelly, A. (2002). Quantification of perfusion using bolus tracking magnetic resonance imaging in stroke: Assumptions, limitations, and potential implications for clinical use. Stroke, 33(4), 1146-1151.

[6] Koh, T.S., Bisdas, S., Koh, D.M., Thng, C.H. (2011). Fundamentals of tracer kinetics for dynamic contrastenhanced MRI. Journal of Magnetic Resonance Imaging, 34(6), 1262-1276.

[7] Sourbron, S.P., Buckley, D.L. (2013). Classic models for dynamic contrast-enhanced MRI. NMR in Biomedicine, 26(8), 1004-1027.

[8] Tofts, P., Brix, G., Buckley, D., et al. (1999). Estimating kinetic parameters from dynamic contrast-enhanced T(1)-weighted MRI of a diffusable tracer: Standardized quantities and symbols. Journal of Magnetic Resonance Imaging, 10(3), 223-232.

[9] Tofts, P.S. (1997). Modeling tracer kinetics in dynamic Gd-DTPA MR imaging. Journal of Magnetic Resonance Imaging, 7(1), 91-101.

[10] Brix, G., Kiessling, F., Lucht, R., Darai, S., Wasser, K., Delorme, S., Griebel, J. (2004). Microcirculation and microvasculature in breast tumors: Pharmacokinetic analysis of dynamic MR image series. Magnetic Resonance in Medicine, 52, 420-429.

[11] St Lawrence, K.S., Lee, T.Y. (1998). An adiabatic approximation to the tissue homogeneity model for water exchange in the brain: I. Theoretical derivation. Journal of Cerebral Blood Flow and Metabolism, 18(12), 13651377.
[12] Koh, T.S., Zeman, V., Darko, J., et al. (2001). The inclusion of capillary distribution in the adiabatic tissue homogeneity model of blood flow. Physics in Medicine and Biology, 46(5), 1519-1538.

[13] Bartoš, M., Jiř́ik, R., Kratochvíla, J., Standara, M. Starčuk Jr., Z., Taxt, T. (2014). The precision of DCEMRI using the tissue homogeneity model with continuous formulation of the perfusion parameters. Magnetic Resonance Imaging, 32(5), 505-513.

[14] Østergaard, L. (2005). Principles of cerebral perfusion imaging by bolus tracking. Journal of Magnetic Resonance Imaging, 22(6), 710-717.

[15] Essig, M., Shiroishi, M.S., Nguyen, T.B., et al. (2013). Perfusion MRI: the five most frequently asked technical questions. American Journal of Roentgenology, 200(1), 24-34.

[16] Donahue, K.M., Krouwer, H.G., Rand, S.D., Pathak, A.P., Marszalkowski, C.S., Censky, S.C., Prost, R.W. (2000). Utility of simultaneously acquired gradientecho and spin-echo cerebral blood volume and morphology maps in brain tumor patients. Magnetic Resonance in Medicine, 43(6), 845-853.

[17] Pike, M.M., Stoops, C.N., Langford, C.P., Akella, N.S., Nabors, L.B., Gillespie, G.Y. (2009). High-resolution longitudinal assessment of flow and permeability in mouse glioma vasculature: Sequential small molecule and SPIO dynamic contrast agent MRI. Magnetic Resonance in Medicine, 61(3), 615-625.

[18] Sourbron, S., Heilmann, M., Biffar, A., Walczak, C., Vautier, J., Volk, A., Peller, M. (2009). Bolus-tracking MRI with a simultaneous T1- and T2*-measurement. Magnetic Resonance in Medicine, 62(3), 672-681.

[19] Schmiedeskamp, H., Andre, J.B., Straka, M., et al. (2013). Simultaneous perfusion and permeability measurements using combined spin- and gradient-echo MRI. Journal of Cerebral Blood Flow and Metabolism, 33(5), 732-743.

[20] Heilmann, M., Walczak, C., Vautier, J., et al. (2007). Simultaneous dynamic $\mathrm{T} 1$ and $\mathrm{T} 2 *$ measurement for AIF assessment combined with DCE MRI in a mouse tumor model. Magnetic Resonance Materials in Physics, Biology and Medicine, 20(4), 193-203.

[21] Sourbron, S., Heilmann, M., Walczak, C., Vautier, J., Schad, L.R., Volk, A. (2013). T2*-relaxivity contrast imaging: First results. Magnetic Resonance in Medicine, 69(5), 1430-1437.

[22] Schmiedeskamp, H., Straka, M., Newbould, R.D., et al. (2012). Combined spin- and gradient-echo perfusionweighted imaging. Magnetic Resonance in Medicine, 68, 30-40.

[23] Lüdemann, L., Warmuth, C., Plotkin, M., Förschler, A., Gutberlet, M., Wust, P., Amthauer, H. (2009). Brain tumor perfusion: Comparison of dynamic contrast enhanced magnetic resonance imaging using $\mathrm{T}_{1}, \mathrm{~T}_{2}$, and $\mathrm{T}_{2}^{*}$ contrast, pulsed arterial spin labeling, and $\mathrm{H} 2(15) \mathrm{O}$ 
positron emission tomography. European Journal of Radiology, 70(3), 465-474.

[24] Macíček, O., Jiřík, R., Bartoš, M., et al. (2013). Interleaved DCE and DSC-MRI. In Trends in Biomedical Engineering, Košice, Slovak Republic: Technical University, 177-180.

[25] Macíček, O., Jiř́ík, R., Bartoš, M., et al. (2013). Interleaved Time-Effective DCE-MRI. Magnetic Resonance Materials in Physics, Biology and Medicine, 26 (Suppl. 1), 204-205.

[26] Kratochvíla, J., Jiř́ik, R., Bartoš, M., Standara, M., Starčuk Jr., Z., Taxt, T. (2016). Distributed capillary adiabatic tissue homogeneity model in parametric multichannel blind AIF estimation using DCE-MRI. Magnetic Resonance in Medicine, 75(3), 1355-1365.

[27] Li, K.-L., Zhu, X. P., Waterton, J., Jackson, A. (2000). Improved 3D quantitative mapping of blood volume and endothelial permeability in brain tumors. Journal of Magnetic Resonance Imaging, 12(2), 347-357.

[28] Parker, G.J.M., Roberts, C., Macdonald, A., et al. (2006). -derived functional form for a populationaveraged high-temporal-resolution arterial input function for dynamic contrast-enhanced MRI. Magnetic Resonance in Medicine, 56(5), 993-1000.

[29] Taxt, T., Jiř́ík, R., Rygh, C.B., et al. (2012). Singlechannel blind estimation of arterial input function and tissue impulse response in DCE-MRI. IEEE Transactions on Biomedical Engineering, 59(4), 1012-1021.

[30] Mouridsen, K., Christensen, S., Gyldensted, L., Østergaard, L. (2006). Automatic selection of arterial input function using cluster analysis. Magnetic Resonance in Medicine, 55(3), 524-531.

[31] Madsen, M.T. (1992). A simplified formulation of the gamma variate function. Physics in Medicine and Biology, 37(7), 1597-1600.

[32] Grüner, R., Bjørnarå, B.T., Moen, G., Taxt, T. (2006). Magnetic resonance brain perfusion imaging with voxel-specific arterial input functions. Journal of Magnetic Resonance Imaging, 23(3), 273-284.

[33] Kershaw, L. E., Buckley, D. L. (2006). Precision in measurements of perfusion and microvascular permeability with T1-weighted dynamic contrast-enhanced MRI. Magnetic Resonance in Medicine, 56(5), 986-992.

[34] Schabel, M. C. (2012). A unified impulse response model for DCE-MRI. Magnetic Resonance in Medicine, 68(5), 1632-1646.

[35] Liu, G., Sobering, G., Duyn, J., Moonen, C.T.W. (1993). A functional MRI technique combining principles of echo-shifting with a train of observations (PRESTO). Magnetic Resonance in Medicine, 30(6), 764-768.

[36] Pedersen, M., Klarhöfer, M., Christensen, S. r., Ouallet, J.-C., Østergaard, L., Dousset, V., Moonen, C. (2004). Quantitative cerebral perfusion using the PRESTO acquisition scheme. Journal of Magnetic Resonance Imaging, 20(6), 930-940.

[37] Boxerman, J.L., Hamberg, L.M., Rosen, B.R., Weisskoff, R.M. (1995). MR contrast due to intravascular magnetic susceptibility perturbations. Magnetic Resonance in Medicine, 34(4), 555-566.

[38] Kjølby, B.F., Østergaard, L., Kiselev, V.G. (2006). Theoretical model of intravascular paramagnetic tracers effect on tissue relaxation. Magnetic Resonance in Medicine, 56(1), 187-197.

[39] Lammertsma, A., Brooks, D.J., Beaney, R.P., et al. (1984). In vivo measurement of regional cerebral haematocrit using positron emission tomography. Journal of Cerebral Blood Flow and Metabolism, 4, 317322.

[40] Šonka, M., Hlaváč, V., Boyle, R. (2008). Image Processing, Analysis, and Machine Vision. Thomson Learning.

[41] Bagher-Ebadian, H., Jain, R., Nejad-Davarani, S.P., et al. (2012). Model selection for DCE-T1 studies in glioblastoma. Magnetic Resonance in Medicine, 68(1), 241-251.

[42] Harrer, J.U., Parker, G.J.M., Haroon, H., et al. (2004). Comparative study of methods for determining vascular permeability and blood volume in human gliomas. Journal of Magnetic Resonance Imaging, 20, 748-757.

[43] Kratochvíla, J., Jiř́k, R., Starčuk Jr., Z., Bartoš, M., Standara, M., Taxt, T. (2016). Using multichannel blind AIF estimation with the DCATH model to allow increased temporal sampling interval in DCE-MRI. Magnetic Resonance Materials in Physics, Biology and Medicine, 29 (Suppl. 1), 288-289.

Received May 02, 2018. Accepted October 29, 2018. 